\title{
Cardiovascular Responses of Type A and Type B Behavior Patterns to Visual Stimulation during Rest, Stress and Recovery
}

\author{
Jeong-Mi Lee ${ }^{1)}$ and Shigeki Watanuki ${ }^{2)}$ \\ 1) Graduate School of Design, Kyushu University \\ 2) Faculty of Design, Kyushu University
}

\begin{abstract}
Differences in the cardiovascular responses of individuals with behavior patterns of Type A and Type B were investigated during rest, stress, and recovery by visual stimulation. Thirty healthy undergraduate and graduate students (mean age: $22.18 \pm 1.44$ years) were categorized as Type A $(\mathrm{N}=14)$, or Type B $(\mathrm{N}=16)$ based on the Kwansei Gakuin's daily life questionnaire. The cardiovascular reactivity of all participants was repetitively monitored for 6 sessions, with each session comprising 3 conditional phases, viz., resting, stress, and post-stress recovery. A gray screen was displayed during resting, displeasure-evoking images were displayed under the stress condition, and video clips of a forest or a control image (a gray screen) were displayed during the recovery condition. When participants were subjected to different stimuli on a 42-inch plasma television screen in each session, electrocardiograms (ECG), impedance cardiograms and the blood pressure (BP) of the respective participants were continuously monitored. According to the results, Type A indicated higher sympathetic reactivity than Type B during resting and under stress. As such, Type A indicated a shorter pre-ejection period (PEP) level during resting and a greater cardiac output (CO) increase under stress than Type B. Furthermore, parasympathetic predominance and parasympathetic antagonism accompanying the enhanced sympathetic activity induced by the unpleasant stress images decreased heart rate (HR) in both Type A and Type B, although the decrease in Type A was relatively meager. Unlike previous studies, the present study demonstrated that Type A indicated more enhanced sympathetic reactivity than Type B in resting physiological arousal levels and visual stimulus-induced stress. J Physiol Anthropol 26(1): 1-8, 2007 http:// www.jstage.jst.go.jp/browse/jpa2

[DOI: 10.2114/jpa2.26.1]
\end{abstract}

Keywords: Type-A behavior pattern, enhanced sympathetic reactivity, cardiovascular response, visual stimulus, rest, stress

\section{Introduction}

Due to the unique characteristics of individuals, humans perceive and process emotional information as well as react with different abilities. In other words, based on individual differences in physical strength, personality, behavioral pattern, intelligence, or mental impairment, humans indicate different physiological, subjective, and behavioral responses to the same emotional stimulus physically, or a phenomenon called polytypism.

Polytypism prevails in humans in response to stress as well; viz., some display stronger physiological responses to the same stress environment than others, although self-consciousness of stress may not be apparent. Those more sensitive individuals display the so-called Type A behavior pattern (Type A), and attention has been focused on the relationship of Type A traits with stress-related diseases (Contrada and Krantz, 1988; Lachar, 1993).

Type A is a concept developed from Friedman and Rosenman's findings (Friedman and Rosenman 1959; 1960); patients with coronary heart disease have specific behavioral dispositions, or traits. Type A individuals display certain traits with the following special features: passionate and explosive in speech (loud voice and quick speech), hypervigilant and hyperactive, impatient and rushed for time, hard-driving and competitive, ambitious, hostile, and aggressive (Hugdahl, 1995; Lachar, 1993; Oishi et al., 1999; Palmero et al., 2001). On the other hand, Type B behavior pattern (Type B) indivisuals are those who exhibit a behavior pattern with a lower intensity/frequency of such traits/features.

According to studies dealing with Type A and Type B (Anderson and Lawler, 1995; Oishi et al., 1999; Ward et al., 1986; Williams et al., 1982), Type A indicates relatively enhanced sympathetic reactivity (i.e. increases in systolic blood pressure (SBP), heart rate (HR) and catecholamine secretion). Furthermore, Type A tends to take a longer time to recover from stress exposure than Type B. As such, the 
accumulation of higher activation/slow recovery from daily stress is probably a contributing factor in rendering Type A susceptible to cardiovascular diseases (Palmero et al., 2001; Smith and Rhodewalt, 1986).

Hitherto, studies dealing with stress-induced physiological responses in Type A have employed tasks that demanded active coping (e.g., public speaking, structured interview, reaction time, mental arithmetic and Stroop color-word interference, etc.), because the behavioral and physiological traits (HR and BP increases) of Type A are especially induced in these active tasks. According to these studies, measures such as BP and HR are useful in differentiating responses between Type A and Type B, and the response consistency of SBP serves especially as a highly reliable variable in monitoring physiological reactivity (reviewed in Contrada et al., 1985; Holmes, 1983; Houston, 1983). For example, Ward et al. (1986) investigated cardiovascular responses in Type A and Type B men by employing 6 tasks (mental arithmetic, hypothesis-testing, video game response, task-reaction time, handgrip, cold pressor). As the result, SBP indicated enhanced physiological reactivity of Type A more consistent than HR and BP by showing significant Type $\mathrm{A} / \mathrm{B}$ differences in the 3 tasks.

However, tasks employed in the laboratory to induce mental stress include passive-stress tasks such as distress film and distress recall as well. While active-stress tasks enhance sympathetic activity, distress film is known to be particularly associated with parasympathetic dominance (Palomba et al., 2000). As such, passive-stress tasks may induce cardiovascular sympathetic responses different from those induced by activestress tasks in Type A and Type B. Furthermore, this difference may also influence the physiological recovery response after stress exposure, suggesting possible discrepancies in poststress recovery between exposure to active- and passive-stress tasks.

Interestingly, findings of 'being healed,' 'getting rest,' 'feeling free from all kinds of obligation,' and/or 'getting rid of stress' have been reported when humans are shown photographs of natural landscapes (Ohta, 2001). Moreover, positive emotions evoked by a visual stimulus (VS) such as a nature film undo the lingering effects of negative emotions (Fredrickson and Levenson, 1998; Fredrickson, 2003). In a study by Fedrikson et al. (2003), anxiety was provoked in a group of participants by asking them to prepare a speech with time constraint before showing them one of four films, eliciting respectively positive emotions of amusement and contentment, no emotion, or sadness. The results indicated that positive emotions improved and facilitated the recovery of anxiety-induced responses (increases in HR, BP, and peripheral vasoconstriction) to baseline values. In this study, a nature-film clip depicting waves breaking on a beach was employed as the visual stimulus to induce contentment.

Furthermore, not only physiological reactivity under stress and during post-stress recovery but also resting physiological reactivity is a potential mechanism that may link behavioral characteristics to cardiovascular diseases (Schroeder et al.,
2000). Although numerous studies on differences in physiological responses between Type A and Type B have employed active-stress tasks focusing on $\mathrm{HR}$ and $\mathrm{BP}$ responses, investigations on the difference of resting physiological reactivity in these two behavioral patterns have been limited.

From the above findings, it is highly possible that the stimulus may affect physiological responses of Type A under stress and during post-stress recovery. In facilitating understanding of physiological polytypism during stress and recovery, it is crucial to elucidate the influences of stress stimulus/task on physiological reactivity in individuals with a certain trait.

Having thus considered the physiological polytypism related to stress and post-stress recovery in humans, we next examined the differences in cardiovascular responses of Type A and Type $\mathrm{B}$ during rest, under stress, and during post-stress recovery with distress images and nature images.

\section{Methods}

\section{Participants}

Thirty healthy undergraduate and graduate students (16 males and 14 females, $22.16 \pm 1.44$ years) participated in this study with written informed consent after having been briefed on the experimental objectives and procedures. Females were restricted to those who were in the follicular phase of menstrual cycle. Type A tendency was assessed by a selfassessment scale of Kwansei Gakuin's daily life questionnaire (KG questionnaire) (Yamasaki et al., 1992), which comprised original items suitable for Japanese adults.

With a score range of $0-88$, the KG questionnaire contains 50 items: 44 items related to and 11 items not related to Type A tendencies. Three subscales complementing the questionnaire are: aggression/hostility (AH) consisting of 18 items; hard-driving lifestyle/time-urgency (HT) 16 items; and speed/power of action (SP) 15 items. The answer for each item is either 'Yes' or 'No' with respective scores of 2 or 0 , while a score of 1 is given to answers with a question mark ('?').

Based on the score distribution, 14 (male: 6, female: 8 ) and 16 (male: 10, female: 6) participants indicating scores above and below the median value (score: 40) were categorized respectively as Type A and Type B. With reference to the score distribution of Type A and Type B (Table 1), scores of Type A for all listed scales were significantly different from those for Type B $(p s<.002)$.

\section{Experimental design and stimuli}

Experiments were conducted in an electrically shielded, sound-attenuated, and temperature-controlled chamber with a room temperature of $28^{\circ} \mathrm{C}$ and relative humidity of $50 \%$. Subjects wore a T-shirt with short pants. To delete any physiological effects induced by the stimulus per se, stimuli of various contents were employed and each participant was repetitively exposed to 6 sessions with each session monitored 
Table 1 The score distribution for Type A and 3 subscales of the KG questionnaire and Type A/B differences in mean scores of the scales

\begin{tabular}{|c|c|c|c|c|c|}
\hline & & \multirow{2}{*}{ Type A score } & \multicolumn{3}{|c|}{ Subscale score } \\
\hline & & & $\begin{array}{l}\text { Aggression/ } \\
\text { Hostility }\end{array}$ & $\begin{array}{l}\text { Hard-driving/ } \\
\text { Time urgency }\end{array}$ & $\begin{array}{l}\text { Speed/ } \\
\text { Power }\end{array}$ \\
\hline $\begin{array}{c}\text { Total } \\
(\mathrm{N}=30)\end{array}$ & $\begin{array}{c}\mathrm{M} \pm \mathrm{SD} \\
\text { Median } \\
\text { Range }\end{array}$ & $\begin{array}{c}39.30 \pm 14.121 \\
40 \\
12-72\end{array}$ & $\begin{array}{c}19.00 \pm 7.344 \\
18 \\
4-36\end{array}$ & $\begin{array}{c}12.13 \pm 6.709 \\
11.5 \\
1-28\end{array}$ & $\begin{array}{c}12.43 \pm 6.185 \\
10 \\
2-26\end{array}$ \\
\hline $\begin{array}{c}\text { Type A } \\
(\mathrm{N}=14)\end{array}$ & $\mathrm{M} \pm \mathrm{SD}$ & $50.00 \pm 11.348$ & $23.29 \pm 5.928$ & $16.50 \pm 5.585$ & $16.07 \pm 6.120$ \\
\hline $\begin{array}{l}\text { Type B } \\
(\mathrm{N}=16)\end{array}$ & $\mathrm{M} \pm \mathrm{SD}$ & $29.94 \pm 8.575$ & $15.25 \pm 6.455$ & $8.31 \pm 5.160$ & $9.25 \pm 4.282$ \\
\hline $\begin{array}{l}\text { Comparison between } \\
\text { Type A and Type B }\end{array}$ & $\begin{array}{c}F(1,28) \\
p\end{array}$ & $\begin{array}{l}30.303 \\
<.001\end{array}$ & $\begin{array}{l}12.479 \\
<.002\end{array}$ & $\begin{array}{l}17.411 \\
<.001\end{array}$ & $\begin{array}{l}12.768 \\
<.002\end{array}$ \\
\hline
\end{tabular}

under 3 conditions: during rest, stress and post-stress recovery. A gray background for the resting condition, displeasureevoking images for the stress condition, and 5 nature-video clips and a gray screen (as a control stimulus) for the recovery condition were presented.

The 5 nature-video clips were silent moving images showing a Japanese forest created by experts: roadside trees in the forest (A), old trees covered with moss (B), a stream in the forest (C), autumnal tints (D), and beech and cedar trees (E).

The stress stimuli were adopted through the result of another group experiment. First of all, 154 images that related to displeasure like disgust were collected from websites and modified to create a strong impact. With a 5-point Likert scale (1: not at all $\sim 5$ : very much), the stress intensity of each image was evaluated in 14 undergraduate and graduate students (male: 8; female: 6). 54 images $(3.348 \pm 1.136)$ were selected from ones with a higher stress intensity, and 6 complete sets $(9$ images per set) of stress stimuli were designed for the experiment. The images of the 6 sets were adjusted such that each set displayed approximately equal stress intensity.

All stimuli were respectively displayed for 3 minutes on a 42-inch plasma television screen located $1.5 \mathrm{~m}$ in front of the participant, who was sitting on a chair. To avoid order effect or carry-over effect by repeated measures, a presentation order of stress and recovery stimuli was counterbalanced with a rotation method, and was altered for each participant.

\section{Data acquisition and analysis}

All cardiovascular signals were continuously recorded for every session.

Electrocardiogram (ECG) signals were monitored with bipolar percordial leads amplified by a bioelectric amplifier (AB-621G; Nihon Kohden, Japan). Using the thoracic electrical impedance method, signals from tape-type electrodes attached on the neck and chest were measured by impedance plethysmogram (AI-601G; Nihon Kohden, Japan). The BP was monitored with a non-invasive continuous BP meter (Jentow 7700; Colin, Japan) using the Tonometry method, where a sensor was attached to the left wrist and a cuff was affixed to the left upper arm. Biosignals were converted to 16-bit using an A/D board (Nihon Santeku Co. Ltd., Japan) before being stored in a personal computer (IBM). The signals were digitized by a sampling rate of $1000 \mathrm{~Hz}$.

Cardiovascular parameters such as heart rate (HR), highfrequency (HF-HRV) and low-frequency of heart rate variability (LF-HRV) and the LF/HF ratio, stroke volume $(\mathrm{SV})$, pre-ejection period (PEP), left ventricular ejection time (LVET), and systolic (SBP) and diastolic BP (DBP) were derived. Cardiac output $(\mathrm{CO}=\mathrm{SV} \times \mathrm{HR})$, mean $\mathrm{BP}$ $(\mathrm{MBP}=(\mathrm{SBP}-\mathrm{DBP}) / 3+\mathrm{DBP})$, and total peripheral resistance $(\mathrm{TPR}=\mathrm{MBP} / \mathrm{CO})$ were then calculated.

The analysis method of HRV is as follows. The 3-min R-R interval data were interpolated at $6 \mathrm{~Hz}$. After applying a Hamming window to the 1024 data points, power spectrum analysis was performed with Fast Fourier Transform. The spectral power at 0.15 to $0.40 \mathrm{~Hz}$ was defined as the $\mathrm{HF}$ component and those at 0.03 to $0.15 \mathrm{~Hz}$ as the LF component. $\mathrm{HF}$ and LF components were expressed in normalized units, which represent the relative value of each power component in proportion to the total power minus the very low-frequency component (0-0.03 Hz).

\section{Statistical analysis}

Software SPSS11.5.1J was employed for statistical analysis. In order to examine the Type A/B differences of physiological responses in the respective conditions, repeated measures analysis of variance was performed; the 6 sessions were treated as a within-subject factor, while the physiological values during resting and the values of physiological changes under stress (value under stress less resting value) and during recovery (recovery value less stress value) were treated as within-subject variables. Type A/B were treated as between- 
$\square$ Type A $\square$ Type B
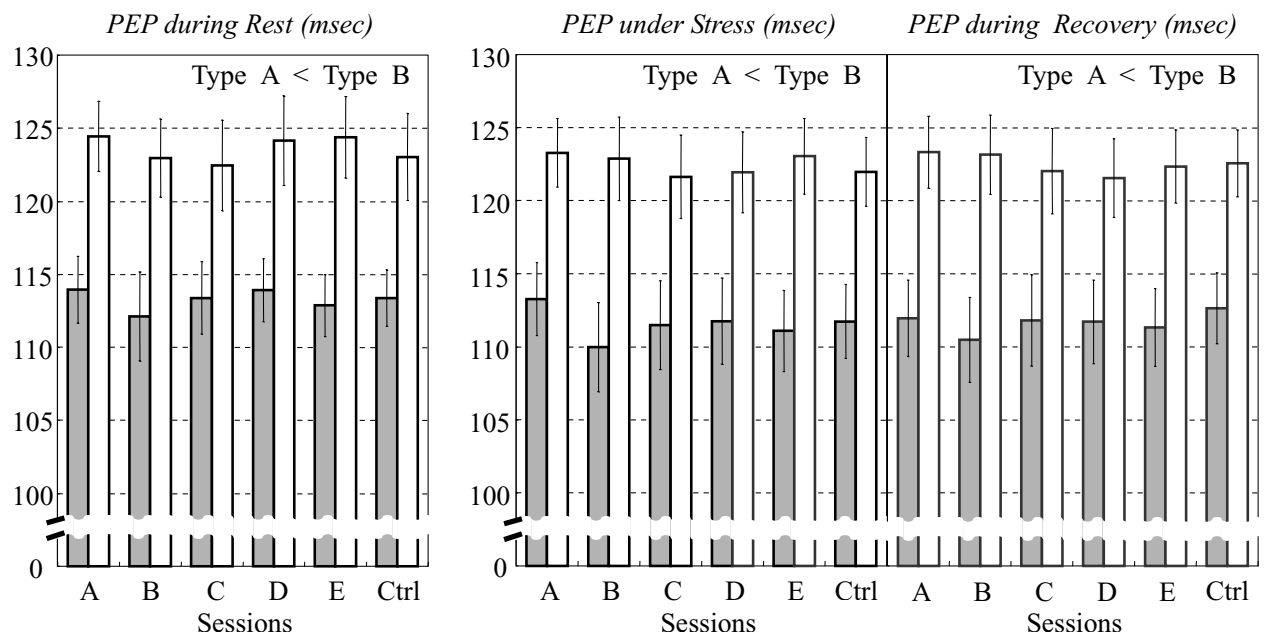

Fig. 1 Pre-ejection period (PEP) values during rest, stress and recovery of Type A and Type B (M \pm SE). PEP in the respective sessions with 5 nature-video clips (A-E) and a control image as a recovery stimulus is shown; roadside trees in the forest (A), old trees covered with moss (B), a stream in the forest (C), autumnal tints (D), beech and cedar trees (E), and gray screen (Ctrl). Type A showed a shorter PEP than Type $\mathrm{B}$ in all sessions and under all conditions.

subject factor. Moreover, in order to examine response changes under stress and recovery conditions according to Type A/B, the paired t-test was used for comparing the stress-resting values, or the recovery-stress values between Type A and Type B. Furthermore, contrast analysis was performed to examine whether the differences in physiological responses to the 5 nature-video clips and gray screen during the recovery condition would interact with the Type A / B factor.

\section{Results}

With regard to Type A scores of Type A, Type B, and total (Type A+B) participants, differences in sex were not significant. In addition, although the repeated measures analysis of variance on physiological responses under the 3 conditions indicated a significant sex effect in resting SBP (male $>$ female) and the LF/HR ratio under stress and during recovery (female $>$ male under stress; male $>$ female during recovery), these sex effects did not affect Type A/B differences. Consequently, Type $\mathrm{A} / \mathrm{B}$ differences were compared as males and females together.

\section{Differences in resting response}

Response differences between Type A and Type B were significant even during rest. With regard to PEP, the main effect of Type $\mathrm{A} / \mathrm{B}$ was significant $(F(1,28)=8.338, p<0.008)$; viz., Type $\mathrm{A}$ indicated a shorter PEP than Type $\mathrm{B}$ in all sessions (Fig. 1). There were no significant main effects of Type A/B in other physiological parameters (HR, LF-HRV, HFHRV, LF/HF ratio, SV, CO, LVET, SBP, DBP, MBP and TPR).

\section{Response differences of the stress condition}

With reference to stress images, although Type A/B differences in SV increases were not significant, those in HR and $\mathrm{CO}$ changes were significant. Although HR decreased in both Type A and Type B, the latter showed a decrease more than that of Type $\mathrm{A}(F(1,28)=5.144, p<0.032)$. Moreover, $\mathrm{CO}$ was significantly increased in Type A only, with changes greater than Type B $(F(1,28)=7.524, p<0.011)$ (Fig. 2). As for DBP, the main effect of Type $\mathrm{A} / \mathrm{B}$ was significant, although Type A showed a decrease greater than that of Type B $(F(1,28)=5.613, p<0.025)$. Although SBP and TPR indicated response patterns similar to DBP, main effects of Type A/B in SBP and TPR were not significant (Fig. 3).

\section{Response differences of recovery condition}

No significant main effects of Type A/B were observed during recovery. Moreover, in results of contrast analysis, interactions between Type $\mathrm{A} / \mathrm{B}$ and recovery stimuli of the nature-video clips and control image were not significant. In other words, recovery response profiles elicited by the naturevideo clips and control image in Type A were not different from those in Type B.

\section{Discussion}

The results of the present study indicated discrepancies from previous studies, viz., differences in resting responses between Type A and Type B, as well as enhanced sympathetic reactivity of Type A under passive stress by the VS.

According to the review of Smith and Rhodewalt (1986), although there is no Type $\mathrm{A} / \mathrm{B}$ difference in resting physiological levels and physiological responses to situations involving minimal psychological challenge or demand, a Type $\mathrm{A} / \mathrm{B}$ difference emerges in physiological responses to situations involving difficult tasks, threats to self-esteem, 
$\square$ Type A $\square$ Type B
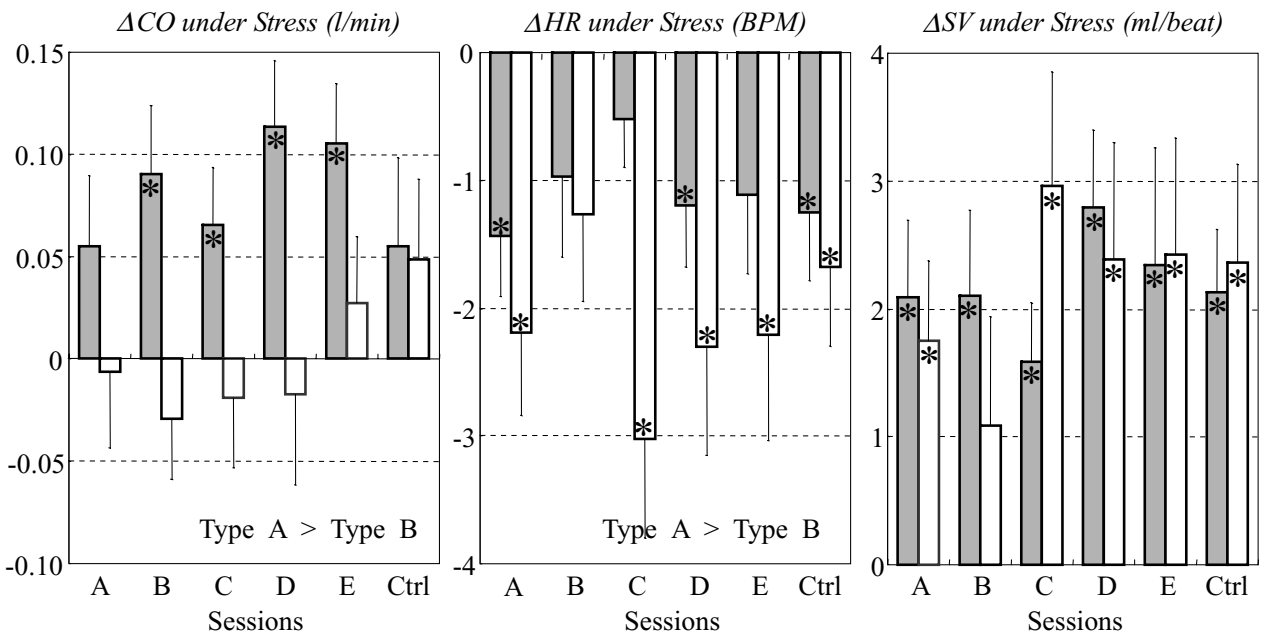

Fig. 2 Changes of cardiac output (CO), heart rate (HR) and stroke volume (SV) in Type A and Type B under stress condition (M \pm SE). HR and $\mathrm{CO}$ displayed the significant main effects of Type A/B, with smaller HR decrease and greater CO increase in Type A. The main effect of Type A/B in SV was not significant. The sessions were classified according to the recovery stimulus in the respective sessions. "*" indicates a statistically significant change compared with the resting value at the $p<.05$ level.

$\square$ Type A $\square$ Type B

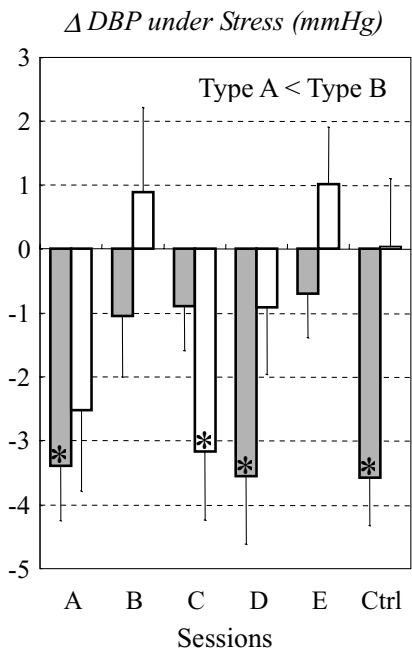

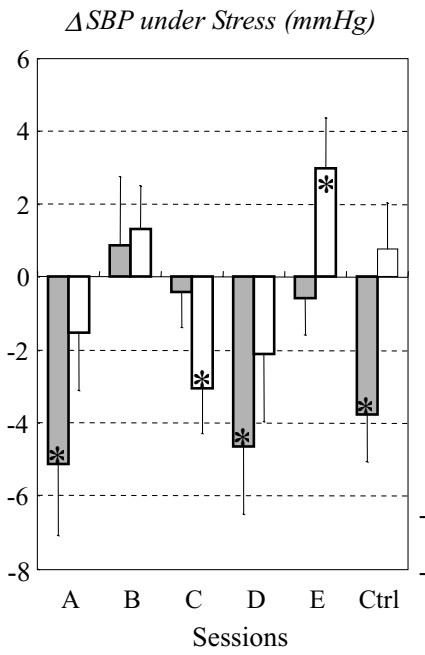

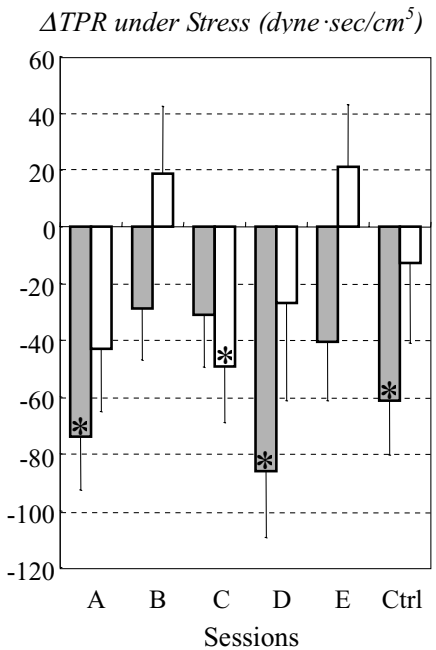

Fig. 3 Changes of diastolic blood pressure (DBP), systolic BP (SBP) and total peripheral resistance (TPR) in Type A and Type B under stress condition $(\mathrm{M} \pm \mathrm{SE}$ ). DBP exhibited the significant main effect of Type $\mathrm{A} / \mathrm{B}$, with significantly greater decreases observed in Type A. Except for session C, SBP and TPR manifested greater decreases (similar to those of DBP) in Type A than Type B without the main effect of Type A/B. The sessions were classified according to the recovery stimulus in the respective sessions. "*" indicates a statistically significant change compared with the resting value at the $p<.05$ level.

threats to control, or negative interpersonal interactions. However, in the present study, Type A showed a significantly shorter PEP than Type B during rest. PEP is an index representing the $\beta$-adrenergic sympathetic activity on ventricular contractility (Sherwood et al., 1990). Therefore, this result suggests that Type A might have a higher resting level of physiological arousal. In fact, PEP values under stress $(F(1,28)=8.886, \quad p<0.006)$ and during recovery $(F(1,28)=9.090, p<0.006)$ also revealed the significant main effects of Type $\mathrm{A} / \mathrm{B}$, consistently manifesting a shorter PEP (Fig. 1).

In general, achievement motivation in Type A individuals is aroused by extrinsic orientation such as interpersonal competition, superiority, and prestige (Sturnam, 1999). Besides, as Type A individuals aspire to perform a task with high achievement levels, and practice cognitive self-regulation of negative self-evaluation and self-criticism with reference to accomplishments, they strive aggressively to reduce the 


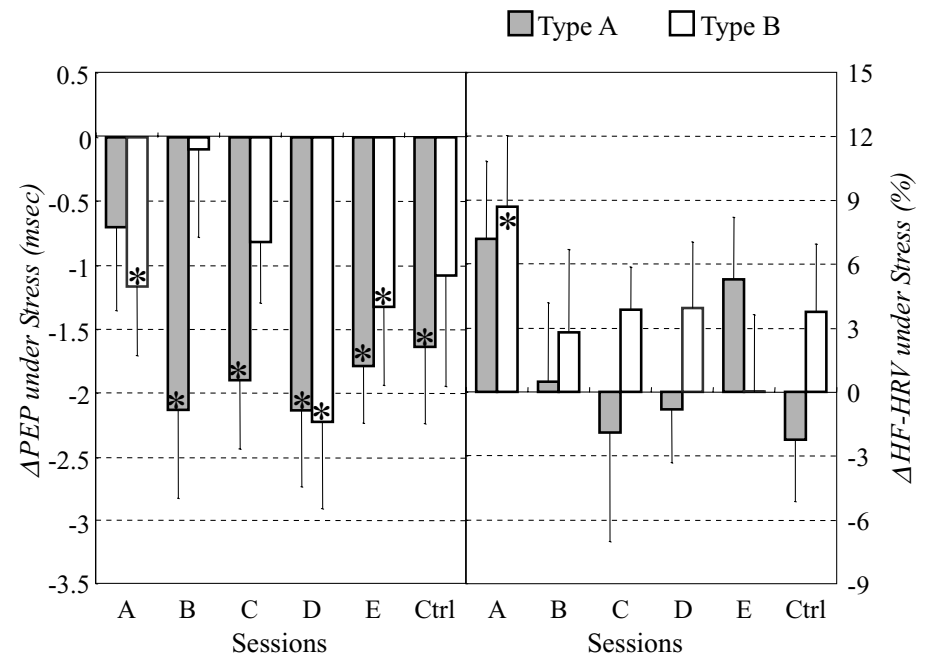

Fig. 4 Changes of pre-ejection period (PEP) and high-frequency component of heart rate variability (HF-HRV) in Type A and Type B under the stress condition $(\mathrm{M} \pm \mathrm{SE})$. PEP decreases were accompanied by HF-HRV increases, and this result indicated concomitant activation of the sympathetic and parasympathetic nerves. There was no main effect of type A/B in PEP and HF-HRV. The sessions were classified according to the recovery stimulus in the respective sessions. "*" indicates a statistically significant change compared with the resting value at the $p<.05$ level.

discrepancy between goals and accomplishments (O'Keeffe and Smith, 1988). As such, hitherto studies related to psychophysiological differences of Type A/B have emphatically employed active-stress tasks such as challenge and social interaction tasks (Houston, 1983). In the present study, however, passive stress was given to the participants using unpleasant VS, and Type A exhibited an increase greater than that of Type B. In short, it is clear that Type A tends to manifest heightened physiological reactivity to a greater extent than Type B, even with passive-stress tasks.

According to the results of the present study, although passive stress with VS induced significant Type A/B differences in HR, DBP and CO, only $\mathrm{CO}$ indicated enhanced sympathetic reactivity of Type A, while HR and DBP were decreased in both Type A and Type B. These findings reflect the results of stimulus- or task-dependent physiological responses and parasympathetic inhibitory responses in reciprocation to increased sympathetic activity.

Emotional VS generally induce specific responses such as sustained cardiac deceleration (HR decrease and/or t-amplitude increase), and this deceleration is more remarkable during the viewing of unpleasant compared with pleasant VS (Lang et al., 1993; Palomba et al., 1997). In addition, this cardiac deceleration observed in passive exposure to unpleasant VS might be interpreted as perceptual-attentional requirements in processing emotional information, and might be associated with parasympathetic dominance (Palomba et al., 2000). Cardiac activities are controlled by dual innervations of the sympathetic and parasympathetic (vagal) nerves, and sympathetic activation is substantially suppressed when vagal activation occurs simultaneously. These predominant inhibitory effects of the vagal nerve become progressively stronger with increasing sympathetic background activity
(Uijtdehaage and Thayer, 2000). Uijtdehaage and Thayer (2000) have demonstrated the vagal predominance on HR control in humans with the use of physical stress. With reference to results of the present study, despite the fact that PEP (most directly influenced by $\beta$-adrenergic sympathetic activity on ventricular contractility) decreased with stress VS, HF-HRV (an index for parasympathetic activity on the heart) increased (Fig. 4).

We may thus interpret the present results as follows. When $\beta$-adrenergic sympathetic activation is induced by unpleasant VS, cardiac contractility increases (PEP decrease and SV increase) with concomitant marked decreases in HR controlled predominantly by the PNS to eventually suppress CO increase. However, as parasympathetic antagonism accompanying sympathetic excitation in Type A is less potent than Type B (Fukudo et al., 1992; Muranaka et al., 1988), the HR decrease in Type A was less than that in Type B, and higher sympathetic reactivity was then observed in Type A with regard to $\mathrm{CO}$. Note that the net result of BP decrease was induced as a result of inhibited $\mathrm{CO}$ increase and TPR decrease (Fig. 2 3).

Previous studies with only SBP, DBP and HR have mainly examined the enhanced sympathetic reactivity on the cardiovascular system in Type A. However, when the multiple hemodynamic parameters related with the blood volume ejected to aorta from the left ventricle by heart contractions in the present study were taken into consideration, enhanced sympathetic reactivity of Type A was observed from the cardiac side (PEP and $\mathrm{CO}$, etc.) and not the vascular side during resting and under stress conditions. Based on this finding, sympathetic reactivity accompanying Type-A tendency is predisposed to influence cardiac activity until blood was ejected from the heart more than the vascular activity. In a similar perspective, Contrada et al. (1991) demonstrated 
significantly lower SBP in Type A than in Type B when anger, fear, and distress are induced by autobiographical emotional imagery tasks. With reference to discrepancies in the results (i.e. higher sympathetic reactivity in Type A), they argued that emotional imaginary tasks did not espouse elements that posed a challenge to or required the outpouring of behavioral and physiological features typical of Type A to adopt a proactive response. As stated above, it is obvious that the task features influence cardiovascular reactivity. However, having used few variables (only SBP, DBP and HR) cannot be overlooked either as another reason for which Contrada and colleagues were not able to confirm the elevated cardiovascular reactivity of Type A.

In previous studies, as Type A manifested high sympathetic reactivity with weak parasympathetic reactivity compared with Type B, it was obvious that post-stress recovery was slower than Type B. However, the physiological recovery responses in the present results indicate no significant differences between Type A and Type B, although they supported the notion that the responses of post-stress recovery may be affected by the features of the stress task (e.g., HR increase was shown as the recovery response since HR decrease was induced by stress VS). In this regard, visual scenes of nature are designed as a universal commercial product to reduce stress and attenuate fatigue, as these instill in humans a pleasant feeling with a sense of relaxation and serenity. Although there is a limit to interpreting results obtained from healthy individuals with Type A and Type B tendencies, the fact that physiological recovery responses by nature-video clips were not different between Type A and Type B suggests that nature images may be useful in normalizing the stress-induced cardiovascular changes in Type A.

\section{Conclusion}

The present study confirmed that Type A showed higher sympathetic reactivity than Type B in the resting physiological arousal level and the cardiovascular responses to VS-induced passive stress (not active stress). These results suggest that the physiological variables attributed to enhanced physiological reactivity of Type A are dependent on the features of the stress task. As such, in investigating the physiological reactivity in terms of individual traits, such as Type $\mathrm{A} / \mathrm{B}$, it is important that response discrepancies in task features are taken into consideration and that the responses during rest, stress, and post-stress recovery are monitored using various physiological parameters.

Acknowledgements This project was supported by Sony PCL., Inc. We are thankful to the Solution Produce Group of Sony PCL for providing us technical expertise and mechanical equipment assistance throughout the study. This study was supported by the 21 st century COE program of Kyushu University.

\section{References}

Anderson SF, Lawler KA (1995) The anger recall interview and cardiovascular reactivity in women: An examination of context and experience. J Psychosom Res 39: 335-343

Contrada RJ, Hilton WF, Glass DC (1991) Effects of emotional imagery on physiological and facial responses in Type A and Type B individuals. J Psychosom Res 35(4/5): 391-397

Contrada RJ, Krantz DS (1988) Stress, reactivity, and Type A behavior: Current status and future directions. Ann Behav Med 10: 64-70

Contrada RJ, Wright RA, Glass DC (1985) Psychophysiologic correlates of Type A behavior: Comments on Houston(1983) and Holmes(1983). J Res Pers 19: 12-30

Fredrickson BL (2003) The Value of Positive Emotions: The emerging science of positive psychology is coming to understand why it's good to feel good. Am Sci 91: 330-335

Fredrickson BL, Levenson RW (1998) Positive emotions speed recovery from the cardiovascular sequelae of negative emotions. Cognition \& Emotion 12(2): 191-220

Friedman M, Rosenman RH (1959) Association of specific overt behavior pattern with blood and cardiovascular findings. JAMA 169: 1286-1296

Friedman M, Rosenman RH (1960) Overt behavior pattern in coronary disease: Detection of overt behavior pattern A in patients with coronary disease by a new psychophysiological procedure. JAMA 173: 1320-1326

Fukudo S, Lane JD, Anderson NB, Kuhn CM, Schanberg SM, McCown N, Muranaka M, Suzuki J, Williams RB Jr (1992) Accentuated vagal antagonism of $\beta$-adrenergic effects on ventricular repolarization: evidence of weaker antagonism in hostile Type A men. Circulation 85: 2045-2053

Holmes DS (1983) An alternative perspective concerning the differential psychophysiological responsivity of persons with the Type A and Type B behavior patterns. J Res Pers 17: $40-47$

Houston BK (1983) Psychophysiological responsivity and the Type A behavior pattern. J Res Pers 17: 22-39

Hugdahl K (1995) Cardiovascular Psychophysiology. In Psychophysiology: The mind-body perspective. Harvard University Press, Cambridge MA, 197-233

Lachar BL (1993) Coronary-prone behavior: Type A behavior revisited. Tex Heart Inst J 20: 143-151

Lane JD, Adcock RA, Burnett RE (1992) Respiratory sinus arrhythmia and cardiovascular responses to stress. Psychophysiology 29(4): 461-470

Lang PJ, Greenwald MK, Bradley MM, Hamm AO (1993) Looking at pictures: affective, facial, visceral, and behavioral reactions. Psychophysiology 30: 261-273

Muranaka M, Lane JD, Suarez EC, Anderson NB, Suzuki J, Williams RB Jr (1988) Stimulus-specific patterns of cardiovascular reactivity in Type A and B subjects: evidence for enhanced vagal reactivity in type B. Psychophysiology 25(3): 330-338

Ohta H (2001) A phenomenological approach to natural 
landscape cognition. J Environ Psychol 21: 387-403

Oishi K, Kamimura M, Nigorikawa T, Nakamiya T, Williams RE, Horvath SM (1999) Individual differences in physiological responses and Type A behavior pattern. Appl Human Sci 18(3): 101-108

O'Keeffe JL, Smith TW (1988) Self-regulation and Type A behavior. J Res Pers 22: 232-251

Palmero F, Diez JL, Asensio AB (2001) Type A behavior pattern today: relevance of the JAS-S Factor to predict heart rate reactivity. Behav Med 27(1): 28-36

Palomba D, Angrilli A, Mini A (1997) Visual evoked potentials, heart rate responses and memory to emotional pictorial stimuli. Int J Psychophysiol 27: 55-67

Palomba D, Sarlo M, Angrilli A, Mini A, Stegagno L (2000) Cardiac responses associated with affective processing of unpleasant film stimuli. Int J Psychophysiol 36: 45-57

Schroeder KE, Narkiewicz K, Kato M, Pesek C, Phillips B, Davison D, Somers VK (2000) Personality type and neural circulatory control. Hypertension 36: 830-833

Sherwood A, Allen MT, Fahrenberg J, Kelsey RM, Lovallo WR, van Doornen LJP (1990) Committee report: Methodological guidelines for impedance cardiography. Psychophysiology 27: 1-23

Smith TW, Rhodewalt F (1986) On states, traits, and processes: a transactional alternative to the individual difference assumptions in Type A behavior and physiological reactivity. J Res Pers 20: 229-251

Sturnam TS (1999) Achievement motivation and Type A behavior as motivational orientations. J Res Pers 33: 189207

Uijtdehaage SHJ, Thayer JF (2000) Accentuated antagonism in the control of human heart rate. Clin Auton Res 10: $107-110$

Ward MM, Chesney MA, Swan GE, Black GW, Parker SD, Rosenman RH (1986) Cardiovascular responses in Type A and Type B men to a series of stressors. J Behav Med 9(1): $43-49$

Williams RB Jr, Lane JD, Kuhn, CM, Melosh W, White AD, Schanberg SM (1982) Type A behavior and elevated physiological and neuroendocrine responses to cognitive tasks. Science 218(4571): 483-485

Yamasaki K, Tanaka Y, Miyata Y (1992) A Type A questionnaire for Japanese adults (KG's Daily Life Questionnaire): its standardization and methods of application. Type A, 3(1): 33-45 [In Japanese]

Received: September 15, 2006

Accepted: October 25, 2006

Correspondence to: Jeong-Mi Lee, Department of Human Living System Design, Graduate School of Design, Kyushu University, 4-9-1 Shiobaru, Minami-ku, Fukuoka, 815-8540, Japan

Phone: 092-553-4543

Fax: 092-553-4543

e-mail: jmlee@gsd.design.kyushu-u.ac.jp 